\title{
Pancreatic tumor margin detection by oblique incidence diffuse reflectance spectroscopy
}

Alejandro Garcia-Uribe, Cheng-Chung Chang, Jun Zou, Bhaskar Banerjee, John Kuczynski, et al.

Alejandro Garcia-Uribe, Cheng-Chung Chang, Jun Zou, Bhaskar Banerjee, John Kuczynski, Lihong V. Wang, "Pancreatic tumor margin detection by oblique incidence diffuse reflectance spectroscopy," Proc. SPIE 7895, Optical Biopsy IX, 78950J (16 February 2011); doi: 10.1117/12.875919

SPIE. Event: SPIE BiOS, 2011, San Francisco, California, United States 


\title{
Pancreatic Tumor Margin Detection by Oblique Incidence Diffuse Reflectance spectroscopy
}

\author{
Alejandro Garcia-Uribe ${ }^{1,2}$, Cheng-Chung Chang ${ }^{2}$, Jun Zou ${ }^{2}$, Bhaskar Banerjee ${ }^{3}$ \\ John Kuczynski ${ }^{3}$ and Lihong V. Wang ${ }^{1}$ \\ ${ }^{1}$ Department of Biomedical Engineering, Optical imaging laboratory, Washington University in St. Louis, \\ One Brookings Drive, St. Louis MO, USA 63030 \\ ${ }^{2}$ Department of Electrical and Computer Engineering, Texas A\&M University, \\ 3128 TAMU, College Station TX, USA 77843 \\ ${ }^{3}$ Department of Gastroenterology and Hepatology, University of Arizona Medical Center \\ 1501 North Campbell Avenue, Tucson AZ, USA 85724
}

\begin{abstract}
In surgical treatment of pancreatic cancers, the effectiveness of the procedures largely depends on the ability to completely and precisely remove the malignant tumors. We present the ex-vivo use of oblique incidence diffuse reflectance spectroscopy (OIRDS) to detect and differentiate normal from neoplastic tissue. An OIRDS probe has been constructed to provide scattering and absorption information of the pancreatic tissue. To reveal the physiological origin of the difference in these optical signatures, the optical scattering coefficients were extracted along the pancreatic duct with 1-cm spacing. Experimental results show that OIDRS was able to successfully determinate the tumor margins based on the higher optical scattering on malignant tissue.
\end{abstract}

Keywords: diffuse reflectance, scattering, spectroscopy, cancer.

\section{INTRODUCTION}

Pancreatic cancer is a malignant neoplasm of the pancreas. It is estimate that in 2010 more than 43,000 people in the United States will be diagnosed with this disease. ${ }^{1}$ Pancreatic cancer often has a poor prognosis, even when diagnosed early. In surgical treatments of malignant tumors, the effectiveness of the treatment procedures largely depends on the ability to completely and precisely remove the malignant tumor tissue. Generally, the tissue in the suspicious regions is first removed and the histological samples are prepared and analyzed. Based on the results of the histology, the next round of tissue removal is planned and performed. A complete surgery would require multiple cycles of tissue removal and analysis, which results in an extremely long procedure. There is an unmet need for a rapid method of determining free tumor margin at surgery that correlates with formal final histology. Recent studies have shown that the optical absorption and scattering properties of human tissues are closely linked to certain critical physiological signatures of their states of malignancy. ${ }^{2-5}$ Oblique incidence diffuse reflectance spectroscopy (OIDRS) is a spectroscopic method, which utilizes a fiber optic sensor probe (coupled with a data acquisition system), to robustly and accurately measuring the diffuse reflectance of inhomogeneous media (e.g., biological tissues) in contact. ${ }^{6}$ Both the optical absorption and scattering properties of the inhomogeneous media can be readily extracted from the measured diffuse reflectance spectra.

\section{EXPERIMENTAL SETUP}

The OIDRS system is shown in Fig. 1. A halogen lamp is used as the light source. An optical multiplexer allows the light delivery through only one source fiber at a time to the area of interest. The diffuse reflectance is collected by the collection optical fibers. The collection fibers are coupled with the imaging spectrograph that generates an optical spectrum for each fiber. The CCD camera collects the spatially resolved diffused reflectance for the wavelength range from 455 to $765 \mathrm{~nm}$. Finally the information is storage in a personal computer for further analysis. The absorption

Optical Biopsy IX, edited by Robert R. Alfano, Stavros G. Demos,

Proc. of SPIE Vol. 7895, 78950J · @ 2011 SPIE · CCC code: 1605-7422/11/\$18 · doi: 10.1117/12.875919

Proc. of SPIE Vol. 7895 78950J-1 
$\left(\mu_{\mathrm{a}}(\lambda)\right)$ and reduced scattering $\left(\mu_{\mathrm{s}}{ }^{\prime}(\lambda)\right)$ coefficients for a particular wavelength can be estimated from the spatially resolved steady-state diffuse reflectance y solving the inverse problem. For the forward model first we used the modified two-source diffusion theory approximation for an oblique incidence source. ${ }^{7,8}$ The resolved steady-state diffuse reflectance a particular wavelength is described by

$$
R(x)=\frac{1}{4 \pi}\left[\frac{\Delta z\left(1+\mu_{e f f} \rho_{1}\right) \exp \left(-\mu_{e f f} \rho_{1}\right)}{\rho_{1}^{3}}+\frac{\left(\Delta z+2 z_{b}\right)\left(1+\mu_{e f f} \rho_{2}\right) \exp \left(-\mu_{e f f} \rho_{2}\right)}{\rho_{2}^{3}}\right]
$$

where $\rho_{1}$ and $\rho_{2}$ are the distances between the point of observation on the sample surface and the point of incidence. $\Delta \mathrm{z}$ is the distance between the virtual boundary and the tissue depth and $\mathrm{z}_{\mathrm{b}}$ is the distance between the virtual boundary and the sample surface. The effective attenuation coefficient $\left(\mu_{e f f}\right)$ is defined by $\mu_{\mathrm{eff}}=\left(\mu_{\mathrm{a}} / D\right)^{1 / 2}$, where $D$ is the diffusion coefficient, $\mu_{a}$ is the absorption coefficient and $\mu_{s}^{\prime}$ is the reduced scattering coefficient. The diffusion coefficient can be calculated by $D=1 /\left[3\left(0.35 \mu_{\mathrm{a}}+\mu_{\mathrm{s}}{ }^{\prime}\right)\right]$. The distance from the point of incidence to the positive point source $d_{s}=3 D$. The shift of the point sources in the $x$ direction $(\Delta x)=d_{\mathrm{s}} \sin \left(\alpha_{t}\right)$ or

$$
\Delta x=\frac{\sin \left(\alpha_{t}\right)}{0.35 \mu_{a}+\mu_{s}{ }^{\prime}}
$$

where $\alpha_{\mathrm{t}}$ is the angle of refraction (Fig. 3). Using simple transformations the absorption and reduced scattering coefficients can be calculated by

$$
\begin{gathered}
\mu_{a}=\frac{\mu_{e f f}^{2} \Delta x}{3 \sin \left(\alpha_{t}\right)} \\
\mu_{s}^{\prime}=\frac{\sin \left(\alpha_{t}\right)}{\Delta x}-0.35 \mu_{a}
\end{gathered}
$$

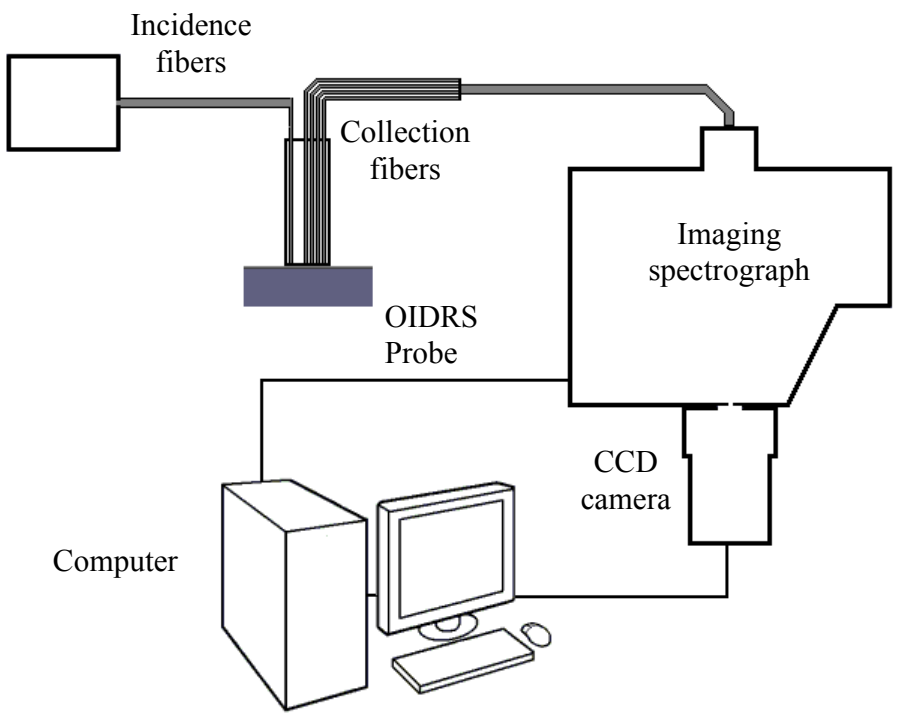

Fig. 1. System setup. 


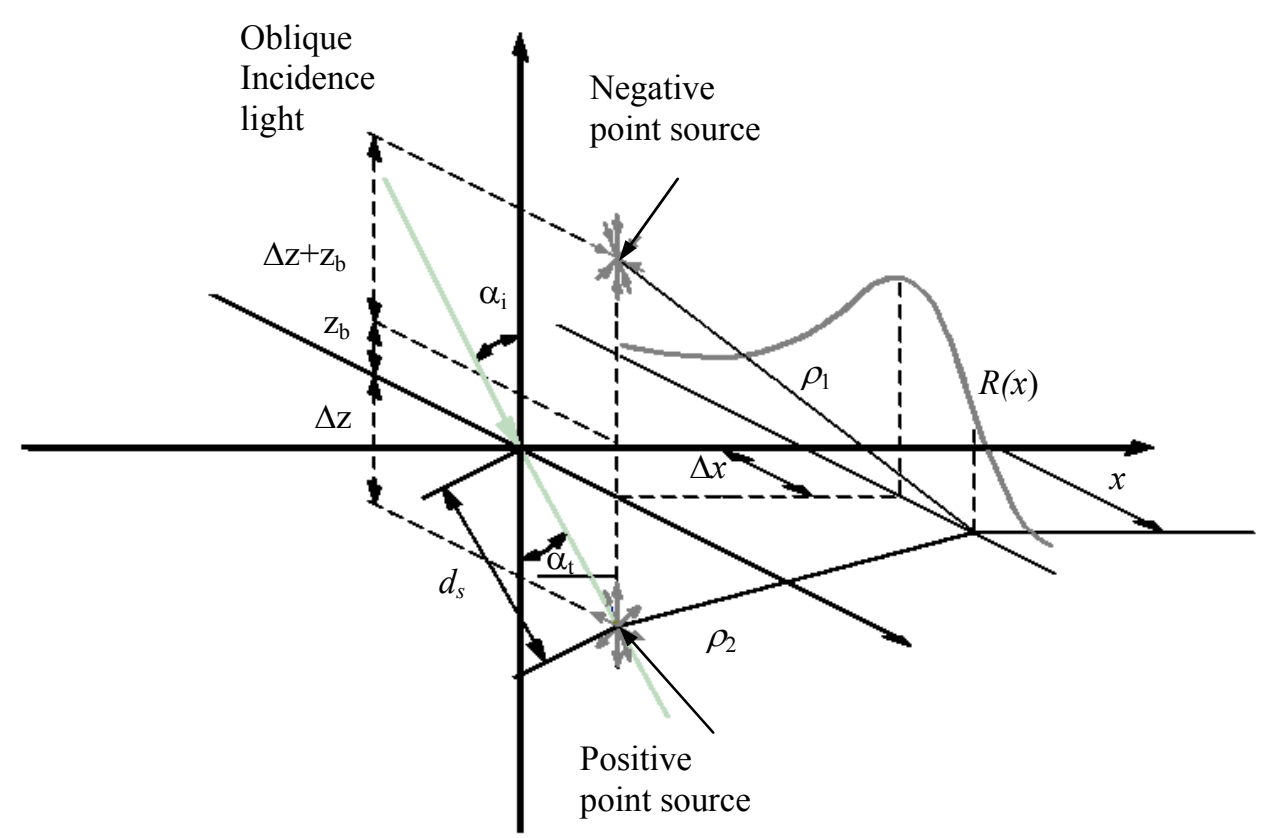

Fig 2. Modified two-source diffusion theory approximation for a single wavelength.

When the distance between the source and the detectors is comparable to the transport mean free path $(\sim 1 \mathrm{~mm})$, diffusion theory does not apply. In this case, scaleable Monte Carlo simulation can be applied to extract optical properties from the diffuse reflectance. ${ }^{9}$ An initial single reference Monte Carlo simulation was conducted with a fixed anisotropy factor $g$ and for reference absorption and scattering coefficients $\left(\mu_{a r}\right.$ and $\left.\mu_{s r}\right)$. The time-resolved diffuse reflectance $R(x, y, t)$ for any new optical parameters, $\mu_{a}$ and $\mu_{s}$, is calculated based on the following relationship

$$
\begin{aligned}
R(x, y, t)=\left(\frac{\mu_{s}}{\mu_{s r}}\right)^{3} R_{r}\left(x \frac{\mu_{s}}{\mu_{s r}}, y \frac{\mu_{s}}{\mu_{s r}}, t \frac{\mu_{s}}{\mu_{s r}}\right) . \\
\cdot \exp \left[-\left(\mu_{a}-\mu_{a r}\right) \frac{\mu_{s}}{\mu_{s r}} c t\right]
\end{aligned}
$$

When distance $(y)$ between any collection fiber and the source fiber is constant, $R(x, t)$ is obtained by first scaling in the $y$ direction. The corresponding steady-state diffuse reflectance is calculated by

$$
R(x)=\int_{0}^{\infty} R(x, t) d t
$$

The inverse problem is solved by calculating and matching the simulated diffuse reflectance results with the actual measurements.

\section{PRELIMINARY EXPERIMENTAL RESULTS}

The OIDRS system was used to measure a pancreatic human sample with cancerous tumor (Fig. 2). Each series of measurements was performed with a 1-cm interval. The OIRDS measurements were later. Previous studies have shown that malignant tissues present different spectroscopic characteristic than their benign counterparts. ${ }^{10-12}$ Malignant tissues usually manifest larger cell density and enlarged cell nuclei. Mitochondria, other cytoplasmic organelles and structures within cell nuclei, are expected to be significant light scatterers and the average effective size of the scattering centers is 
on the order of a few hundred nanometers. ${ }^{13-15}$ Therefore, the relative value of the scattering parameter could serve as a good indicator to differentiate the malignant tissues from the normal ones.

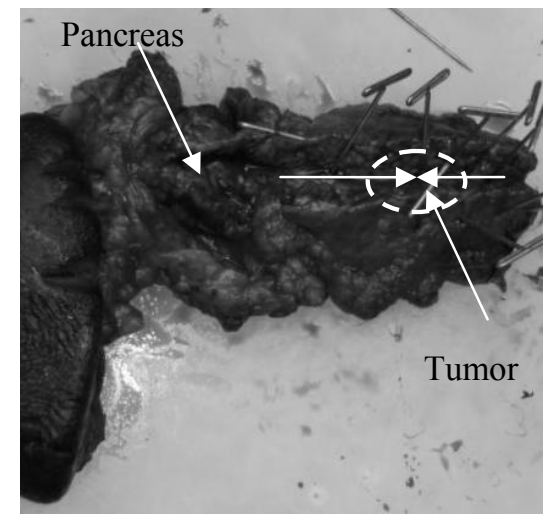

Fig. 2. Human pancreas with a malignant tumor.

Fig. 3 shows the averaged scattering over the entire spectral range for different locations along a single pancreatic sample with adenocarcinoma. The reduced scattering coefficient increases in the region of the cancer. This result matches well with the histological reading of the pancreas specimen.

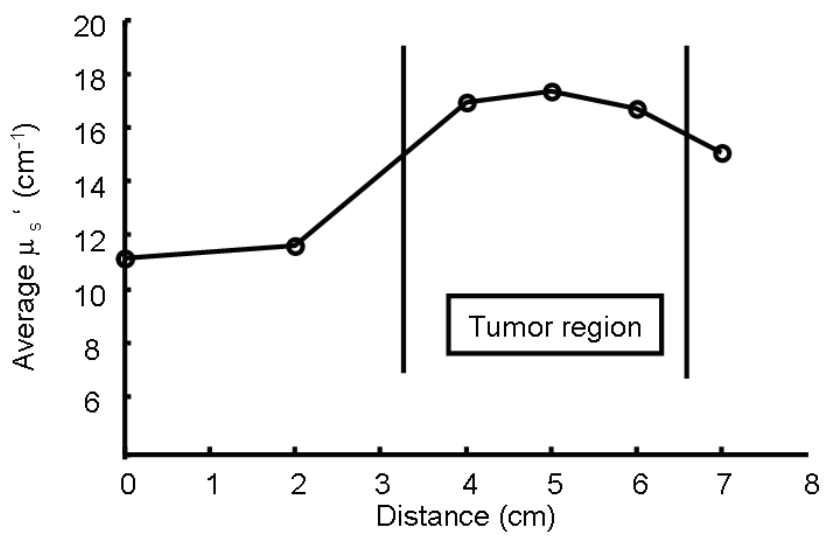

Fig. 3. Average scattering coefficient along the pancreatic sample

\section{CONCLUSIONS}

Our preliminary results show the utility of OIDRS to determinate free tumor margin at surgery. OIDRS is a rapid process that is sensitive to light scattering properties and predicts the presence of cancer. This approach can potentially be used in other operative settings, including surgery for bile duct, gastroesophageal, and head \& neck cancer, to guide accurate but effective tumor removal.

\section{ACKNOWLEDGMENTS}

The authors thank Dr. Andrew Rouse from the University of Arizona Health Sciences Center, for his help during the data collection. This project was sponsored by National Institute of Health grant R01 CA106728. 


\section{REFERENCES}

1. Pancreatic Cancer: National Cancer Institute, http://www.cancer.gov/cancertopics/types/pancreatic (2010).

2. Troy T. L., Page D. L., Sevick-Muraca E. M., "Optical properties of normal and diseased breast tissues: prognosis for optical mammography," Journal of Biomedical Optics 1, 342-355 (1996).

3. Garcia-Uribe A., Kehtarnavaz N., Marquez G, Prieto V, Duvic M, and Wang L. V., "Skin cancer detection by spectroscopic oblique-incidence reflectometry: classification and physiological origins," Applied Optics 43, 2643-2650 (2004).

4. Bargo P. R., Prahl S. A., Goodell T. T., Sleven R. A., Koval G., Blair G., Jacques S. L., "In vivo determination of optical properties of normal and tumor tissue with white light reflectance and an empirical light transport model during endoscopy," J. Biomedical Optics 10, 034018 (2005).

5. Georgakoudi I., Jacobson B. C., Van Dam J., Backman V., Wallace M. B., Muller M. G., Zhang Q., Badizadegan K., Sun D., Thomas G. A., Perelman L. T., Feld M. S., "Fluorescence, reflectance, and light-scattering spectroscopy for evaluating dysplasia in patients with Barrett's esophagus", Gastroenterology 120, 1620-1629 (2001).

6. Garcia-Uribe A., Balareddy K. C., Zou J. and Wang L. V., "Micromachined Fiber Optical Sensor for In-Vivo Measurement of Optical Properties of Human Skin," IEEE Sensors Journal 8, 1698-1703 (2008).

7. Wang L. H. and Jacques S. L., "Use of a laser beam with an oblique angle of incidence to measure the reduced scattering coefficient of a turbid medium," Applied Optics 34, 2362-2366 (1995).

8. Marquez G. and Wang L.-H., "White light oblique incidence reflectometer for measuring absorption and reduced scattering spectra of tissue-like turbid media," Optics Express 1, 454-460 (1997).

9. Farrell T. J. and Patterson M. S., "A diffusion theory model of spatially resolved, steady-state diffuse reflectance for the noninvasive determination of tissue optical properties in vivo," Medical Physics 19, 879-888 (1992).

10. Müller M. G., Valdez T. A., Georgakoudi I., et. al., "Spectroscopic detection and evaluation of morphologic and biochemical changes in early human oral carcinoma," Cancer 97, 1681-1692 (2003).

11. Perelman L. T., Backman V., Wallace M., Zonios G., et al. "Observation of Periodic Fine Structure in Reflectance from Biological Tissue: A New Technique for Measuring Nuclear Size Distribution," Physical Review Letters 80, 627-630 (1998).

12. Weber C. R., Schwarz R. A., Atkinson E. N., Cox D. D., MacAulay C., Follen M., and Richards-Kortum R., "Model-based analysis of reflectance and fluorescence spectra for in vivo detection of cervical dysplasia and cancer," Journal of Biomedical Optics 13, 064016 (2008).

13. Mourant J. R., Freyer J. P., Hielscher A. H., Eick A. A., Shen D., and Johnson T. M., "Mechanisms of Light Scattering from Biological Cells Relevant to Noninvasive Optical-Tissue Diagnostics," Applied Optics 37, 3586-3593 (1998).

14. Mourant J. R., Canpolat M., Brocker C., Esponda-Ramos O., Johnson T. M., Matanock A., Stetter K., and Freyer J. P., "Light scattering from cells: the contribution of the nucleus and the effects of proliferative status," Journal of Biomedical Optics 5, $131-137(2000)$

15. Drezek R., Dunn A., and Richards-Kortum R., "Light scattering from cells: finite-difference time-domain simulations and goniometric measurements," Applied Optics 38, 3651-3661 (1999). 